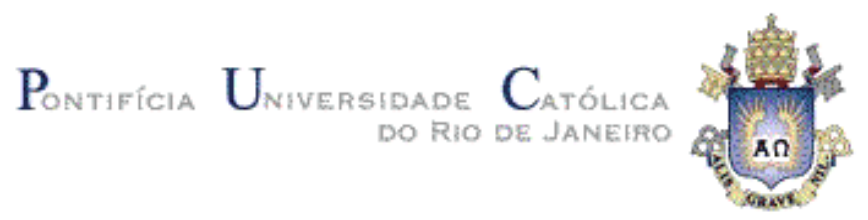

Cassia Daniele dos Santos Silva

\begin{abstract}
Análise do Impacto dos Erros de Previsão no Processo de Planejamento de Produção de uma Empresa Petrolífera.
\end{abstract}

\footnotetext{
Dissertação de Mestrado (Opção profissional)

Dissertação apresentada como requisito parcial para obtenção do título de Mestre pelo Programa de PósGraduação em Engenharia Industrial da PUC-Rio.
}

Orientador: Prof. Carlos Patrício Samanez Co-orientador: Dr. Fabiano Mezadre Pompermayer 


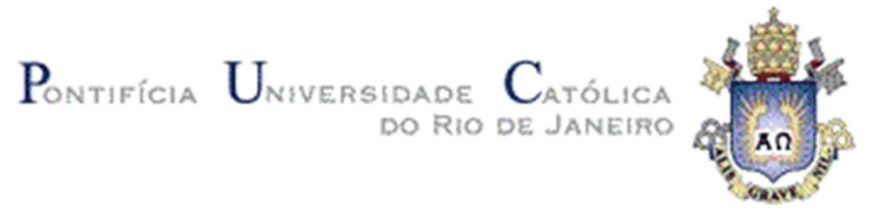

Cassia Daniele dos Santos Silva

\title{
Análise do Impacto dos Erros de Previsão no Processo de Planejamento de Produção de uma Empresa Petrolífera
}

\begin{abstract}
Dissertação apresentada como requisito parcial para obtenção do título de Mestre pelo Programa de PósGraduação em Engenharia Industrial da PUC-Rio. Aprovada pela Comissão Examinadora abaixo assinada.
\end{abstract}

Prof. Carlos Patrício Samanez Orientador e Presidente Departamento de Engenharia Industrial - PUC-Rio

Prof. Fabiano Mezadre Pompermayer Co-orientador Consultor Autônomo

Prof. José Eugênio Leal Departamento de Engenharia Industrial - PUC-Rio

Prof. Marcelo Maciel Monteiro Petróleo Brasileiro - Rio de Janeiro - Matriz

Prof. José Eugênio Leal Coordenador Setorial do Centro Técnico Científico - PUC-Rio 
Todos os direitos reservados. É proibida a reprodução total ou parcial do trabalho sem autorização da universidade, da autora e do orientador.

\section{Cassia Daniele dos Santos Silva}

Engenheira Química, formada pela Universidade Federal Fluminense, com mestrado em tecnologia de processos químicos e bioquímicos, pela Universidade Federal do Rio de Janeiro. É Engenheira de Processamento da Petrobras.

Ficha Catalográfica

Silva, Cassia Daniele dos Santos

Análise do impacto dos erros de previsão no processo de planejamento de produção de uma empresa petrolífera / Cassia Daniele dos Santos Silva ; orientador: Carlos Patrício Samanez ; co-orientador: Fabiano Mezadre Pompermayer. - 2012.

109 f. : il. (color.) ; $30 \mathrm{~cm}$

Dissertação (mestrado)-Pontifícia Universidade Católica do Rio de Janeiro, Departamento de Engenharia Industrial, 2012.

Incluí referências bibliográficas.

1. Engenharia Industrial - Teses. 2. Planejamento integrado de vendas e operações. 3. Cadeia de suprimento de petróleo. 4. Previsão de demanda. 5. Planejamento operacional. 6. Logística. I. Samanez, Carlos Patrício. II. Pompermayer, Fabiano Mezadre. III. Pontifícia Universidade Católica do Rio de Janeiro. Departamento de Engenharia Industrial. IV. Título.

CDD: 658.5 


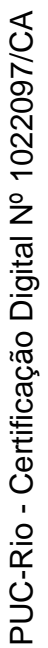

Aos meus pais 


\section{Agradecimentos}

A Deus, por tudo;

Aos meus pais, Eugenio e Vilma, pelo carinho, apoio, compreensão e incentivo;

Ao meu noivo, Marcos, pelo incentivo, paciência e compreensão nos períodos de minha ausência;

Aos meus orientadores, Fabiano Mezadre Pompermayer e Carlos Patrício Samanez, pelo incentivo, estímulo e contribuições nesse estudo;

A Petrobras, pelo apoio geral;

E a todos que me incentivaram e que de alguma forma contribuíram para a realização desta dissertação 


\section{Resumo}

Silva, Cassia Daniele dos Santos; Samanez, Carlos Patrício; Pompermayer, Fabiano Mezadre. Análise do Impacto dos Erros de Previsão no Processo de Planejamento de Produção de uma Empresa Petrolífera. Rio de janeiro, 2012. 109p. Dissertação de Mestrado (Opção profissional) Departamento de Engenharia Industrial, Pontifícia Universidade Católica do Rio de Janeiro.

A necessidade de integração entre os vários componentes de uma cadeia de suprimento e o S\&OP (Sales and Operations Planning) são conceitos amplamente conhecidos pelas empresas, entretanto muitas vezes é difícil alinhar os conceitos teóricos às necessidades e aos processos reais das empresas. O foco deste trabalho é o planejamento operacional da cadeia logística de abastecimento de petróleo e derivados de uma empresa petrolífera. A empresa utiliza um modelo de programação linear determinístico para elaboração do plano. Foram monitorados os desvios entre o realizado e o planejado de diversos parâmetros que influenciam no plano, como preços internacionais, volume de produção de petróleo, demanda de alguns derivados e disponibilidades de unidades de refinaria. Após análise desses desvios, utilizou-se o modelo de programação linear da empresa para elaborar uma série de sensibilidades, retroalimentando o modelo, com a utilização dos erros médios das variáveis. Por fim são agrupadas as informações sobre realizado x planejado x sensibilidade (plano modificado) à incerteza. Os resultados mostram que o plano modificado considerando à incerteza das variáveis através dos erros médios históricos possibilita um planejamento mais robusto, onde o resultado deixa de ser um valor ótimo determinístico e se apresenta como uma faixa de valores bons.

\section{Palavras-chave}

Planejamento integrado de vendas e operações; cadeia de suprimento de petróleo; previsão de demanda; planejamento operacional; logística. 


\section{Abstract}

Silva, Cassia Daniele dos Santos; Samanez, Carlos Patrício (Advisor); Pompermayer, Fabiano Mezadre (Co-Advisor). Analysis of the Forecast Errors Impact in the Process of Production Planning in an Oil Company. Rio de janeiro, 2012. 109p. MSc. Dissertation - Departamento de Engenharia Industrial, Pontifícia Universidade Católica do Rio de Janeiro.

The need for integration between the various components of the supply chain and the S\&OP (Sales and Operations Planning) are concepts widely known by the companies, however it is often difficult to align theoretical concepts to the real needs and processes of companies. The focus of this work is the operational planning of the logistics supply chain of petroleum and derivatives of an oil company. The company uses a deterministic linear programming model for development of the plan. The parameters' deviations between real and planning data, which influence the plan, as international prices, volume of oil production, demand for some oil derivatives and availability of refinery units were monitored. After analyzing of these deviations, we used the linear programming model of the company to develop a range of sensitivities, feeding back the model, using the mean errors of the variables. Finally the information of real $\mathrm{x}$ plan $\mathrm{x}$ modified plan (planned sensitivity) with uncertainty are grouped. The results show that the modified plan considering the uncertainty of the variables through the historical average errors enables a more robust planning, where the result is no longer a deterministic optimal value and it presents itself as a good range of values.

\section{Keywords}

S\&OP; Oil Supply Chain; Demand Forecasting; Operational Planning; Logistics. 


\section{Sumário}

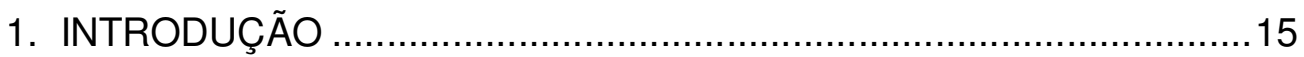

1.1 MOTIVAÇÃO

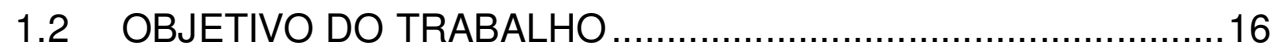

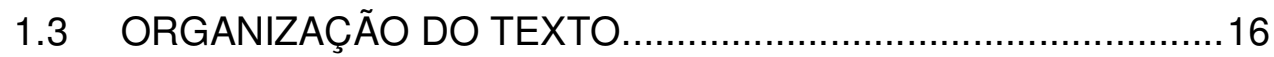

2. REVISÃO BIBLIOGRÁFICA - S\&OP - Planejamento integrado de

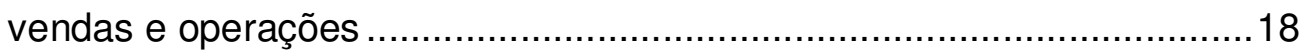

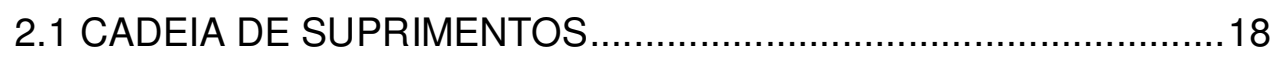

2.2 RISCOS NA CADEIA DE SUPRIMENTOS ……….......................19

2.3 S\&OP - PLANEJAMENTO INTEGRADO DE VENDAS E

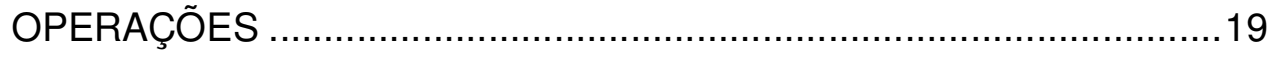

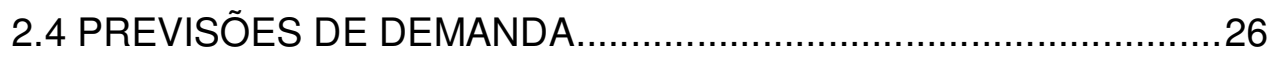

2.5 MÉTODOS DE OTIMIZAÇÃO .........................................................

3. CADEIA DE SUPRIMENTO DE PETROLEO E O PROCESSO DE

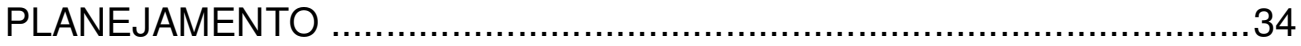

3.1 CADEIA DE SUPRIMENTO DE PETROLEO ................................34

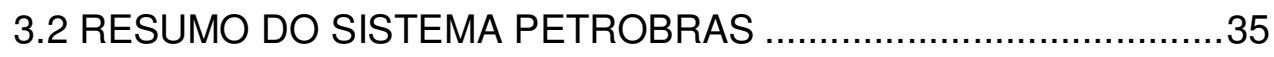

3.3 ASPECTOS POLÍTICOS E PREÇOS DOS COMBUSTÍVEIS .........40

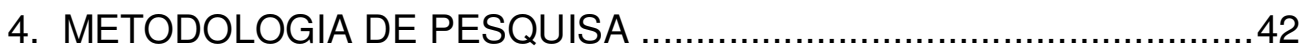

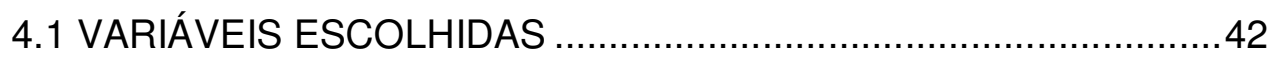

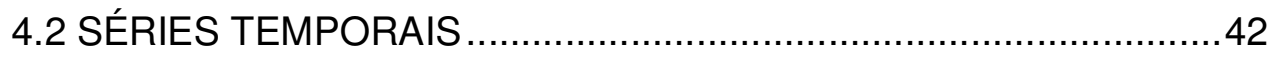

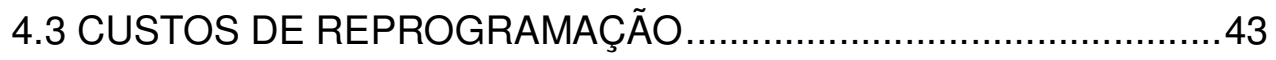

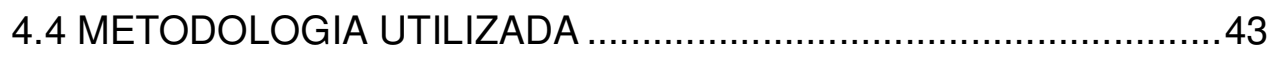

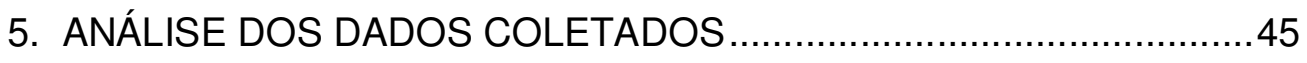

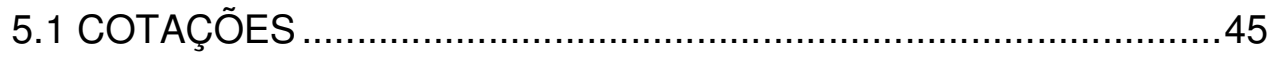

5.2 PRODUÇÃO NACIONAL DE PETRÓLEO ……….........................50

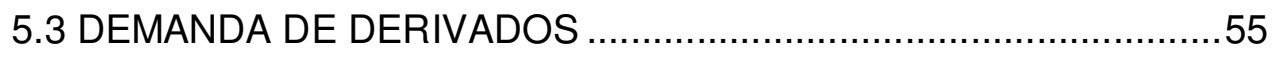

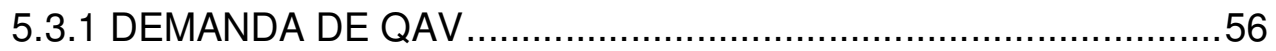

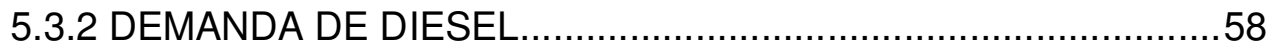

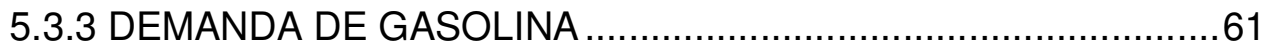

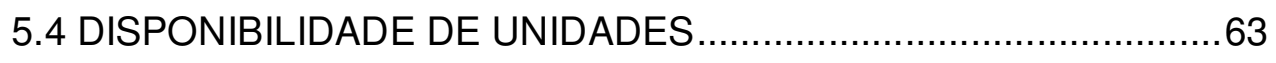

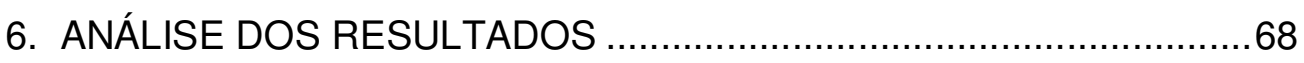

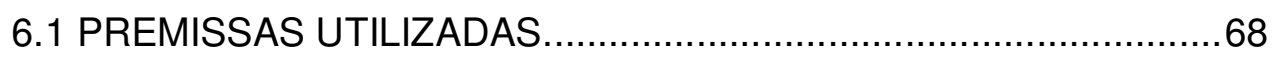


6.2 SENSIBILIDADE AOS ERROS DE PREVISÃO. 69

6.2.1 COTAÇÃO DE PETRÓLEO. 69

6.2.2 PRODUÇÃO DE PETROLEO ……………………...................

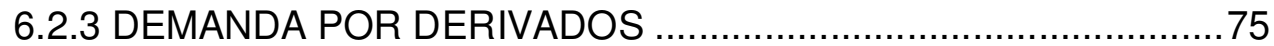

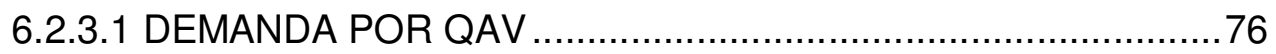

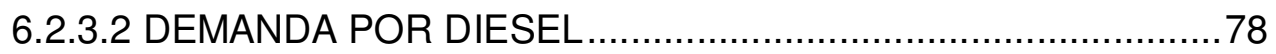

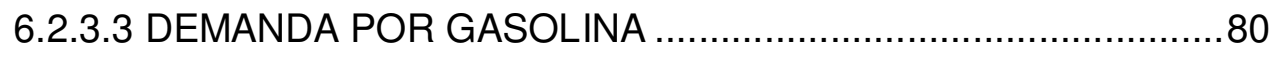

6.2.4 DISPONIBILIDADE DE REFINO (PARADAS DE UNIDADES) ....81

6.3 COMPARAÇÃO ENTRE REALIZADO, PLANEJADO E

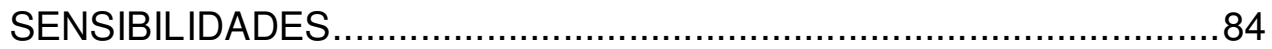

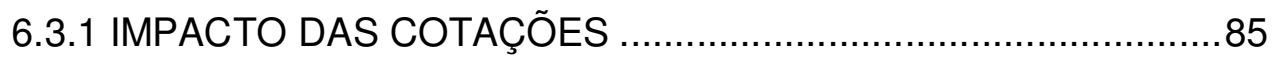

6.3.2 IMPACTO DA PRODUÇÃO DE PETRÓLEO..............................87

6.3.3 IMPACTO DA DEMANDA POR DERIVADOS ……....................90

6.3.4 IMPACTO DA DISPONIBILIDADE DE UNIDADES DE

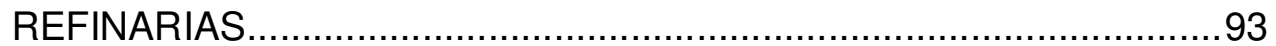

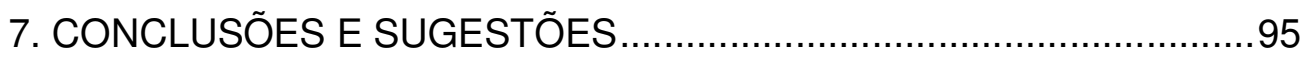

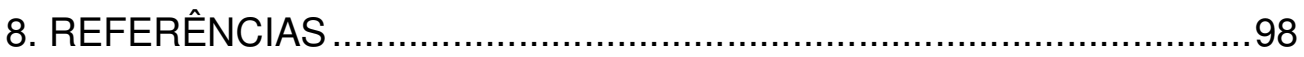

9. APÊNDICES

9.1 DADOS DE PRODUÇÃO NACIONAL ANALISADOS....................100

9.2 DADOS DE MERCADO ANALISADOS ........................................102 
Índice de ilustrações

Figura 1 - Demanda agregada $x$ demanda dos componentes..................21

Figura 2 - Desenho esquemático de refino - Fonte: (ANP, 2012).............37

Figura 3 - Situação real x modelo matemático ………............................38

Figura 4 - Algumas variáveis de entrada e de saída do modelo...............38

Figura 5 - Representação simplificada do Modelo Planab.......................39

Figura 6 - Cotação Petróleo Brent e erros de previsão. …………….......45

Figura 7 - Cotação petróleo WTI e erros de previsão...............................46

Figura 8 - Cotações QAV e erros de previsão ........................................47

Figura 9 - Cotações Diesel e erros de previsão .....................................47

Figura 10 - Cotações nafta e erros de previsão ......................................48

Figura 11- Cotação gasolina e erros de previsão....................................49

Figura 12 - cotações óleo combustível e erros de previsão ......................49

Figura 13 - Produção de petróleo nacional total ...................................51

Figura 14 - Produção de petróleos exportáveis ......................................52

Figura 15 - Produção de petróleo - área VIII ..........................................53

Figura 16 - Produção de petróleo - área $V$..............................................53

Figura 17 - Produção de petróleo - área VI .........................................54

Figura 18- Mercado total de QAV ......................................................56

Figura 19 - Número de decolagens por natureza da operação nos 10 aeroportos mais movimentados- 2010 ................................................57

Figura 20 - Total de passageiros pagos transportado por aeroporto -

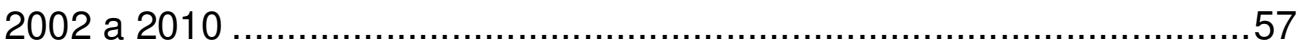

Figura 21 - Mercado de QAV em SP + Bsb .......................................

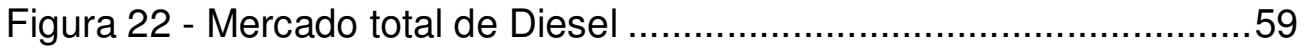

Figura 23 - Mercado de Diesel - Região do Osbra .................................59

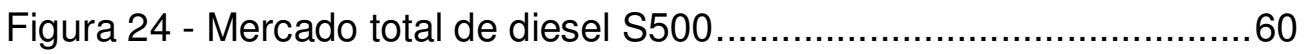

Figura 25 - Mercado total de gasolina ...................................................61

Figura 26 - Influência do preço de etanol na demanda de gasolina .........62

Figura 27 - Mercado de gasolina - área SP ...........................................63

Figura 28 - Disponibilidade de unidades de destilação ............................64

Figura 29 - Disponibilidade de unidades de coqueamento .......................65

Figura 30 - Disponibilidade de unidades de craqueamento catalítico ......66

Figura 31 - Disponibilidade de unidades de hidrotratamento ...................67

Figura 32 - Sensibilidade do resultado econômico do modelo às cotações de petróleo (Número de ocorrências para cada variação obtida)..............70

Figura 33 - Impacto da variação dos preços internacionais, no volume de exportação de petróleo

Figura 34 - Impacto da variação dos preços internacionais, no volume ae

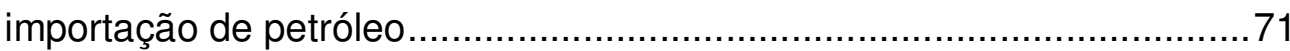

Figura 35 - Sensibilidade do resultado econômico do modelo ao volume de produção próprio de petróleo (Número de ocorrências para cada variação obtida). 
Figura 36 - Impacto da variação da produção de petróleo, no volume

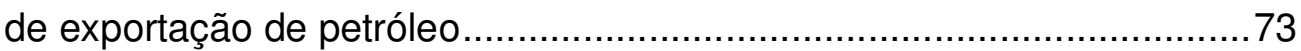

Figura 37 - Impacto da variação da produção de petróleo, no volume de importação de petróleo

Figura 38 - Sensibilidade do resultado econômico do modelo à demanda do mercado interno (Número de ocorrências para cada variação obtida).

Figura 39 - Sensibilidade do resultado econômico do modelo à demanda por QAV (Número de ocorrências para cada variação obtida).

Figura 40 - Impacto da variação da demanda de QAV, no volume de importação de QAV

Figura 41 - Sensibilidade do resultado econômico do modelo à demanda por diesel (Número de ocorrências para cada variação obtida).

Figura 42 - Impacto da variação da demanda de diesel, no volume de importação de diesel

Figura 43 - Sensibilidade do resultado econômico do modelo à demanda por gasolina (Número de ocorrências para cada variação obtida).

Figura 44 - Sensibilidade do resultado econômico do modelo à disponibilidade de unidades (Número de ocorrências para cada variação obtida).

Figura 45 - Impacto da disponibilidade de unidades no volume de

exportação de petróleo.

Figura 46 - Impacto da disponibilidade de unidades no volume de importação de petróleo.

Figura 47 - Impacto da disponibilidade de unidades no volume de importação de derivados

Figura 48 - Sensibilidade da receita de exportação de petróleo às cotações

Figura 49 - Sensibilidade do custo de importação de petróleo às cotações

Figura 50 - Sensibilidade do volume de importação de petróleo às cotações

Figura 51 - Sensibilidade da receita de exportação de petróleo à Produção de Petróleo.

Figura 52 - Sensibilidade do custo de importação de petróleo à

Produção de Petróleo.

Figura 53 - Sensibilidade do volume de exportação de petróleo à

Produção de Petróleo. .90

Figura 54 - Sensibilidade do custo de importação de derivados à demanda de Diesel 
Figura 55 - Sensibilidade do custo de importação de derivados à demanda de gasolina.

Figura 56 - Sensibilidade do volume de importação de diesel à demanda de Diesel

Figura 57 - Sensibilidade do custo de importação de petróleo à disponibilidade de unidades 93

Figura 58 - Sensibilidade do custo de importação de derivados à disponibilidade de unidades. .94

Figura 59 - Produção de petróleo - área I...........................................100

Figura 60 - Produção de petróleo - área II...........................................100

Figura 61 - Produção de petróleo - área III...........................................101

Figura 62 - Produção de petróleo - área IV .........................................101

Figura 63 - Produção de petróleo - área VII .........................................102

Figura 64 - Produção de petróleo - área IX ……………......................102

Figura 65 - Mercado de QAV - região NE............................................103

Figura 66 - Mercado de QAV - região Norte.......................................103

Figura 67 - Mercado de QAV - região RJ-MG-ES ………....................104

Figura 68 - Mercado de QAV - região Sul ........................................104

Figura 69 - Mercado de Diesel - região NE ......................................105

Figura 70 - Mercado de Diesel - região Norte .....................................105

Figura 71 - Mercado de Diesel - região RJ-MG-ES .............................106

Figura 72 - Mercado de Diesel - região Sul ........................................106

Figura 73 - Mercado de Diesel S50 ..............................................107

Figura 74 - Mercado de Gasolina - região NE ...................................107

Figura 75 - Mercado de Gasolina - região Norte ...................................108

Figura 76 - Mercado de Gasolina - região RJ-MG-ES............................108

Figura 77 - Mercado de Gasolina - região Sul ......................................109 
Índice de tabelas

Tabela 1 - Fontes de incerteza na cadeia de suprimento - Fonte:

(Schlegel \& Murray, 2010)...........................................................25

Tabela 2 - Usos da previsão - Fonte: (Kerber \& Deckshage, 2011) .......28

Tabela 3 - Dados consolidados das cotações …………........................50

Tabela 4 - Dados consolidados de produção de petróleo..........................55

Tabela 5 - Dados consolidados de disponibilidade de unidade ................67 


\section{Lista de siglas e abreviaturas}

FCC - Fluid cracking catalytic - Craqueamento catalítico fluido

HDT - Hidrotratamento

EPAM - Erro percentual absoluto médio

QAV - Querosene de Aviação

EM - Erro médio

EAM - Erro absoluto médio

OSBRA - oleoduto com origem em São Paulo e destino em Brasília

ANP - Agencia Nacional do Petróleo

ANAC - Agencia Nacional de Aviação Civil

ÚNICA - União da Indústria de Cana-de-açúcar

S\&OP - Sales and Operations Planning - Planejamento de vendas e operações

PCP - Planejamento e Controle da Produção

CFPR - Collaborative Planning, Forecasting and Replenishment - Planejamento

Colaborativo de Previsão e Reposição

LGN - Líquido de Gás Natural

GLP - Gás liquefeito de Petróleo

WTI - West Texas Intermediate

EIA - Energy Information Administration

APICS - Advancing Productivity, Innovation and Competitive Success - refere-se à "The Associations for Operations Management" 